\title{
REPRESENTING VARIETIES OF ALGEBRAS BY AI,GEBRAS
}

\author{
WAI.TF,R D. NEUMANN \\ (Received 6 Februarv 1969) \\ Dedicated to my father on his 60th birthday \\ Communicated by G. B. Preston
}

\section{Introduction}

In this paper we describe a wav of representing varieties of algebras by algebras. That is, to each variety of algebras we assign an algebra of a certain type, such thai two varieties are rationallv equivalent if and only if the assigned algebras are isomorphic.

Various methods of representing varieties by other objects are known. The earliest seems to be the clones of Philip Hall, described in [1] chapter 3 . A pretty representation of varieties by semigroups with operators, using braids, is due to E. C. Dale [2], see also [6]. We mention also the algebraic theories of Lawvere and I.inton. which represent varieties by certain enriched categories of sets ([4], [5]).

All these methods have something in common, namely in each case the class of representing obiects is in a natural way a category, and the categories one obtains in this way are essentially the same lat least if one only represents varieties defined by finitary operations). In fact it follows from the results of [3] that the morphisms of representing objects correspond precisely to set preserving functors in the opposite direction between the varieties. That is. the category of representing objects is equivalent to the dual of the category of represented varieties and set preserving functors between these varieties. (We shall not worry unduely about the logical difficulties inherent in talking here of a class of classes, as many wavs are known of avoiding them.)

It turns out that if one allows varieties to have operations of countably infinite rank, then the dual of the category of varieties and underlying set preserving functors between varieties is equivalent to the category of a variety of algebras. These algebras by which we represent varieties are a slight modification of Philip Hall's clones. We call them $\boldsymbol{\aleph}_{0}$-clones.

Everything except theorem 3 in this paper carries through with only 
formal changes if one replaces $\boldsymbol{\aleph}_{0}$ by any infinite cardinal number or by the number 1 . Theorem 3 works for infinite regular cardinals.

Since in Linton's terminology an "algebraic theory" of rank $\mathfrak{n}$ ( $\mathfrak{n}$ a regular cardinal) is one whose variety of algebras is definable by operations of rank $<\mathfrak{n}$, our main theorem may also be formulated as: the category of varietal algebraic theories of rank $\leqq \aleph_{1}$ is equivalent to the category of the variety of $\boldsymbol{\aleph}_{\mathbf{0}}$-clones.

\section{The representation}

In the following variety always means variety defined by operations of at most countably infinite rank. To avoid nuisance with the empty algebra we make the convention that it be included in a variety $\mathfrak{A}$ if and only if it occurs as intersection of non-empty subalgebras of some algebra in $\mathfrak{A}$. Thus even if one defines the variety of groups for example by right division alone, the empty group is not allowed.

We recall the definition of rational equivalence. Let $\mathfrak{A}$ and $\mathfrak{B}$ be varieties. Then a map $\Phi$ from $\mathfrak{A}$ to $\mathfrak{B}$ is equational $[3]$ if there exist terms in the defining operations of $\mathfrak{A}$, such that for each $A \in \mathfrak{A}$ the sets underlying $A$ and $\Phi(A)$ are equal and the operations of $\Phi(A)$ are just the operations induced in $A$ by the given $\mathfrak{A}$-terms. A rational equivalence is a bijective equational map whose inverse is also equational. Examples of rational equivalence are boolean lattices with bcolean rings, and the various ways of defining groups by operations and laws.

By Felscher [3], the equational maps from $\mathfrak{A}$ to $\mathfrak{B}$ are precisely the functors from $\mathfrak{A}$ to $\mathfrak{B}$ which preserve underlying sets. Mal'cev's theorem on rational equivalence is of course a special case of this.

Now let $\mathfrak{A}$ be a variety of algebras. We denote by $H(\mathfrak{A})$ the set of $\mathfrak{A}$-algebraic operations of rank $\boldsymbol{\aleph}_{0}$. The following description of $H(\mathfrak{U})$ suffices for our purposes.

Let $X$ be a standard alphabet of letters $p_{1}, p_{2}, \cdots$, and let $F(X, \mathfrak{A})$ be the free $\mathfrak{A}$-algebra with basis $X$. Then the elements of $F(X, \mathfrak{A})$ can be interpreted in the well known way as algebraic operations of rank $\boldsymbol{\aleph}_{\mathbf{0}}$. For example if $\mathbb{B S}$ is the variety of groups, then the element $f=p_{2} p_{5}^{-1} p_{3} \in$ $F(X, B)$ defines the algebraic operation $f\left(a_{1}, a_{2} \cdots\right)=a_{2} a_{5}^{-1} a_{3}$, where $a_{1}, a_{2}, \cdots$ are any elements of any group $A$. In general if $f \in F(X, \mathfrak{A})$; $A \in \mathfrak{A} ; a_{1}, a_{2}, \cdots \in A$; then $f\left(a_{1}, a_{2}, \cdots\right)$ is defined as $\varphi(f)$, where $\varphi$ is the homomorphism from $F(X, \mathfrak{A})$ to $A$ with $\varphi\left(p_{i}\right)=a_{i}$ for each $i$. It is important to note that we consider this as an operation of rank $\boldsymbol{\aleph}_{0}$, even though it may only depend on finitely many of its arguments.

We can hence identify $H(\mathfrak{U})$ with $F(X, \mathfrak{X})$ as a set. The elements 
$p_{1}, p_{2}, \cdots$ of $H(\mathfrak{Q})$ are the trivial operations.

If $f \in H(\mathfrak{X})$ we can apply $f$ to $F(X, \mathfrak{A})$ and form $f\left(f_{1}, f_{2}, \cdots\right)$ for $f_{1}, f_{2}, \cdots \in F(X, \mathfrak{U})$. As an operation $f\left(f_{1}, f_{2}, \cdots\right)$ is defined by

$$
f\left(f_{1}, f_{2}, \cdots\right)\left(a_{1}, a_{2}, \cdots\right)=f\left(f_{1}\left(a_{1}, a_{2}, \cdots\right), f_{2}\left(a_{1}, a_{2}, \cdots\right), \cdots\right) .
$$

Insteari of $f\left(f_{1}, f_{2}, \cdots\right)$ we write $\left(f f_{1} f_{2} \cdots\right)$ and consider $(\cdots)$ as an operation of rank $\boldsymbol{N}_{0}$ on $H(\mathfrak{U})$. We consider $p_{1}, p_{2}, \cdots$ as nullary operations (constants) on $H(\mathfrak{U})$.

Definition. The $\boldsymbol{\aleph}_{0}$-clone $H(\mathcal{Q})$ of the variety $\mathfrak{A}$ is the algebra of $\mathfrak{A}$-algebraic operations of rank $\boldsymbol{\aleph}_{0}$, with algebraic structure given by the $\boldsymbol{N}_{0}$-ary operation $(\cdots)$ and the nullary operations $p_{1}, p_{2}, \cdots$.

$H(\mathfrak{Q})$ clearly satisfies the laws

$\mathrm{C} 2$

$$
\left(x p_{1} p_{2} \cdots\right)=x
$$

C3

$$
\begin{aligned}
\left(p_{i} x_{1} x_{2} \cdots\right) & =x_{i} \quad(i=1,2, \cdots), \\
\left(\left(x y_{1} y_{2} \cdots\right) z_{1} z_{2} \cdots\right) & =\left(x\left(y_{1} z_{1} z_{2} \cdots\right)\left(y_{2} z_{1} z_{2} \cdots\right) \cdots\right) .
\end{aligned}
$$

Definition. An (abstract) $\boldsymbol{N}_{9}$-clone is an algebra defined by an $\boldsymbol{N}_{0}$-ary operation $(\cdots)$ and nullary operations $p_{1}, p_{2}, \cdots$, satisfying the law $\mathrm{C} 1, \mathrm{C} 2$, and $\mathrm{C} 3$.

TheOrem 1. Every abstract $\boldsymbol{N}_{n}$-clone is the $\boldsymbol{\aleph}_{0}$-clone of a variety. Tro varieties have isomorthic $\boldsymbol{N}_{0}$-clones if and only if they are rationally equivalent.

PROOF. If $K$ is an abstract $\boldsymbol{\aleph}_{0}$-clone, define to each $k \in K$ an operation of rank $\aleph_{0}$ on $K$ by

$$
\dot{f}_{k}\left(k_{1}, k_{2}, \cdots\right)=\left(k k_{1} k_{2} \cdots\right) .
$$

Denote the algebra with set $K$ and these operations $\dot{f}_{k}$ by $F(K)$. We claim $F(K)$ is relatively free with basis $X=\left\{p_{1}, p_{2}, \cdots\right\}$. Indeed, firstly it is clear that $X$ generates $F(K)$, for if $k \in K$ then $k=t_{k}\left(p_{1}, p_{2}, \cdots\right)$ by C1. Secondly any map of $X$ into $F(K)$ extends to an endomorphism of $F(K)$, for if $\varphi: X \rightarrow F(K)$ is any map, define $\bar{\varphi}: F(K) \rightarrow F(K)$ by

$$
\bar{\varphi}(k)=f_{k}\left(\varphi\left(p_{1}\right), \varphi\left(p_{2}\right), \cdots\right)
$$

for $k \in K$. C2 and $\mathrm{C} 3$ give respectively that $\bar{\varphi} \mid X==\varphi$ and that $\bar{\varphi}$ is an endomorphism of $F(K)$.

Hence $F(K)$ is the free algebra on basis $X$ of the generated variety $\operatorname{QSP}(F(K))$.

Definition. The variety of $K$-modules $\mathfrak{B}(K)$ is defined to be $\mathfrak{B}(K)=Q S P(F(K))$.

Since we can identify $F(K)$ with $F(X, \mathfrak{B}(K))$, it is immediate from the definitions that $H(\mathfrak{B}(K))=K$ as $\boldsymbol{N}_{0}$-clones. 
Furthermore for any variety $\mathfrak{A}$, the transition from $F(X, \mathfrak{A})$ to $F(H(\mathfrak{A}))=F(X, \mathfrak{B}(H(\mathfrak{A})))$ is given by replacing the defining operations of $F(X, \mathfrak{A})$ by all algebraic operations of rank $\boldsymbol{\aleph}_{n}$ on $F(X, \mathfrak{A})$. This does not alter the rational equivalence type, and since a variety defined by at most countably infinitary operations is already determined by its free algebra of rank $\boldsymbol{\aleph}_{0}, \mathfrak{A}$ is rationally equivalent to $\mathfrak{B}(H(\mathfrak{U}))$.

Thus the transitions $\mathfrak{B}$ from $\aleph_{n}$-clones to varieties and $H$ from varieties to $\boldsymbol{\aleph}_{0}$-clones are mutually inverse (up to rational eouivalence of varieties), so the thenrem follows.

We have seen that every variety $\mathfrak{A}$ is, up to rational equivalence, a variety of $K$-modules for some (unioue up to isomorphism) $\boldsymbol{\aleph}_{n}$-clone $K$. $K$ is the set of $\mathfrak{A}$-algebraic operations of rank $\mathfrak{\aleph}_{n}$, so every $\mathfrak{A}$-algebra is given by the action of $K$ on a set. Compare this with the case of varieties defined by at most unary operations. It is well known that such a variety is rationally equivalent to a variety of $T$-modules for some monoid $T$. In fact this is precisely what one obtains if one does everything for the cardinal number 1 instead of $\aleph_{0}$. Thus "1-clones" are simply monoids.

We now come to the theorem promised in the introduction. Let $\mathbb{E}$ be the category of $\boldsymbol{\aleph}_{0}$-clones and homorphisms, $\mathscr{V}$ ar the category of varieties and underlving set preserving functors between varieties.

THEOREM 2. $\mathfrak{B}: \mathbb{E} \rightarrow \mathscr{V}$ ar and $H: \mathscr{V}$ ar $\rightarrow \mathbb{C}$ are in a natural way contravariant functors. and they detine an equivalence of the category $\mathbb{E}$ with the dual category $\mathscr{V}$ ar* of $\mathscr{V}$ ar.

In view of the previous discussion, this theorem is essentially the thenrem of Felscher on equational maps (loc. cit.). We therefore only define the functors, and omit the proof that they do what is required.

Suppose $\varphi: K \rightarrow L$ is a homomorphism of $\boldsymbol{\aleph}_{n}$-clones. For any $L$-module $A$ there is a natural $K$-module structure on $A$, namely for $k \in K$ and $a_{1}, a_{2}, \cdots \in A$ define $k\left(a_{1}, a_{2}, \cdots\right)$ to be $\varphi(k)\left(a_{1}, a_{2}, \cdots\right)$. This defines a functor $\mathfrak{B}(p): \mathfrak{B}(L) \rightarrow \mathfrak{B}(K)$, and by construction it preserves underlying sets. Thus $\mathfrak{B}$ is a contravariant functor from $\mathbb{S}$ to $\mathscr{V}$ ar.

Conversely if $\Phi: \mathfrak{A} \rightarrow \mathfrak{B}$ is a set preserving functor of varieties, then the map of $F(X, \mathfrak{B})$ into its "universal $\mathfrak{A}$-algebra" (given by the front adjunction belonging to the adjoint functor to $\Phi$ ) gives a map $F(X, \mathfrak{B}) \rightarrow F(X, \mathfrak{A})$. This is compatible with the $\boldsymbol{\aleph}_{0}$-clone structure, so it defines a homomorphism $H(\Phi): H(\mathfrak{B}) \rightarrow H(\mathfrak{X})$. Thus $H$ is a contravariant functor from $\mathscr{V}$ ar to $\mathfrak{E}$.

One is often only interested in varieties defined by finitary operations. For want of a better place we insert the following theorem here, which is an immediate consequence of theorem 2 in [7]. 
Theorem 3. The variety $\mathfrak{A}$ is definable by couniably many finitary operaitions if and only if $H(\mathfrak{A})$ is couniable.

\section{Examples and discussion}

Let $\mathfrak{U}$ be a variety, and $f$ an $\mathfrak{A}$-operation of finite rank $n$. If we consider $f$ as an operation of rank $\boldsymbol{\aleph}_{0}$, we need a law or laws which say that $f$ only depends on $n$ of its arguments. For $n \geqq 1$ a law with this effect is

$$
f\left(x_{1}, x_{2}, \cdots, x_{n}, x_{1}, x_{1}, \cdots\right)=f\left(x_{1}, x_{2}, \cdots, x_{n}, x_{n+1}, \cdots\right),
$$

for (1) says that $f$ does not depend on what is substituted for $x_{n+1}, x_{n+2}, \cdots$. For $n=0$ one can use

$$
f\left(x_{1}, x_{3}, x_{5}, \cdots\right)=f\left(x_{2}, x_{4}, x_{6}, \cdots\right) .
$$

In $H(\mathfrak{Q})$ (1) and (2) become

$$
\begin{aligned}
& \left(f p_{1} p_{2} \cdots p_{n} p_{1} p_{1} \cdots\right)=f, \\
& \left(f p_{1} p_{3} p_{5} \cdots\right)=\left(f p_{2} p_{4} p_{6} \cdots\right) .
\end{aligned}
$$

EXAMPLE 1. Let $\&$ be the variety of groups, $G=H(\leftrightarrow)$ the corresponding $\boldsymbol{\aleph}_{0}$-clone. $G$ is the $\boldsymbol{\aleph}_{0}$-clone generated by three elements $m, i$, and $e$ subject to the relations

$$
\begin{aligned}
& \left(m p_{1} p_{2} p_{1} p_{1} \cdots\right)=m \\
& \left(i p_{1} p_{1} p_{1} p_{1} \cdots\right)=i \\
& \left(e p_{1} p_{3} p_{5} p_{7} \cdots\right)=\left(e p_{2} p_{4} p_{6} \cdots\right) \\
& \left(m m p_{3} p_{1} p_{1} \cdots\right)=\left(m p_{1}\left(m p_{2} p_{3} p_{1} p_{1} \cdots\right) p_{1} p_{1} \cdots\right) \\
& \left(m p_{1} i p_{1} p_{1} \cdots\right)=e \\
& \left(m p_{1} e p_{1} p_{1} \cdots\right)=p_{1} .
\end{aligned}
$$

Indeed $(3),(4),(5)$ state respectively that $m$ is binary, $i$ unary, and $e$ constant; (6) is the associative law

$$
m\left(m\left(x_{1}, x_{2}, \cdots\right), x_{3}, \cdots\right)=m\left(x_{1}, m\left(x_{2}, x_{3}, \cdots\right), \cdots\right) ;
$$

(7) is the inverse law $m\left(x_{1}, i\left(x_{1}, \cdots\right), \cdots\right)=e$; and (8) is the identity law $m\left(x_{1}, e, \cdots\right)=x_{1}$.

EXAMPLE 2. The $\boldsymbol{\aleph}_{0}$-clone $G_{a}$ of the variety $\mathscr{G}_{a}$ of abelian groups is obtained from $G$ by adding the relation

$$
\left(m p_{2} p_{1} p_{1} \cdots\right)=m \text {. }
$$

The obvious epimorphism $G \rightarrow G_{a}$ corresponds (via theorem 2) to the inclusion of $\mathfrak{S S}_{\boldsymbol{a}}$ in $\mathbb{G}$. 
EXAMPLE 3. The $\boldsymbol{\aleph}_{0}$-clone $M$ of the variety $\mathfrak{M}$ of monoids is generated by two elements $m$ and $e$ satisfying relations (3), (5), (6), and (8). The obvious homomorphism $M \rightarrow G$ is injective, and corresponds to the "forget functor" (i) $\rightarrow \mathfrak{M}$ which assigns to each group the underlying multiplicative monoid.

EXAmple 4 . The $\boldsymbol{\aleph}_{0}$-clone $S$ containing only the elements $p_{1}, p_{2}, \cdots$ corresponds to the variety $\subseteq$ of algebras with no non-trivial operations -that is sets. For any non-singular $\boldsymbol{\aleph}_{0}$-clone $K$ the inclusion $S \subseteq K$ corresponds to the underlying set functor $\mathfrak{B}(K) \rightarrow S$.

In general it is clear that a surjective homomorphism $K \rightarrow L$ of $\boldsymbol{\aleph}_{0}$-clones, since it is just an "addition of laws" to $\mathfrak{B}(K)$, corresponds to an inclusion of $\mathfrak{B}(L)$ as a subvariety of $\mathfrak{B}(K)$. Similarly an injective homomorphism $K \rightarrow L$ corresponds to a pure forget functor from $\mathfrak{B}(L)$ to $\mathfrak{B}(K)$ (that is a set preserving functor $\Phi: \mathfrak{B}(L) \rightarrow \mathfrak{B}(K)$ such that $Q S P \Phi(\mathfrak{B}(L))=$ $\mathfrak{B}(K)$ ). Since the monos in $\mathbb{E}$ are just the injective homorphisms (this holds in any variety), the pure forget functors are precisely the epis in $\mathscr{V}$ ar. However the monos in $\mathscr{V}$ ar turn out to be the injective functors, which includes more than just the inclusions of subvarieties. For instance the pure forget functor from groups to monoids is injective, since a group structure is determined by its underlying monoid structure, so this functor is both mono and epi in $\mathscr{V}$ ar.

EXAMPLE 5. Consider the variety of semigroups which satisfy the laws $x x=x$ and $x y z=x z$ (these laws are equivalent to the single law $x y x=x)$. The corresponding $\boldsymbol{\aleph}_{0}$-clone is generated by an element $m$ subject to $(3),(6)$, and

$$
\begin{aligned}
& \left(m p_{1} p_{1} \cdots\right)=p_{1} \\
& \left(m m p_{3} \cdots\right)=\left(n p_{1} p_{3} \cdots\right) .
\end{aligned}
$$

One verifies easily that this $\boldsymbol{\aleph}_{0}$-clone is isomorphic to $S \times S$ by an isomorphism which maps $m$ onto the element $\left(p_{1}, p_{2}\right) \in S \times S$. Since product in the category $\mathbb{C}$ of $\boldsymbol{\aleph}_{0}$-clones corresponds to sum in the category $\mathscr{V}$ ar, we may denote this variety of semigroups by $\widetilde{s}+\widetilde{ }$.

More generally the sum $\mathfrak{A}+\mathfrak{B}$ in the category $\mathscr{V}$ ar is given by $\mathfrak{A}+\mathfrak{B}=\mathfrak{B}(H(\mathfrak{U}) \times H(\mathfrak{B}))$. The projections of $H(\mathfrak{U}) \times H(\mathfrak{B})$ onto $H(\mathfrak{U})$ and $H(\mathfrak{B})$ correspond to embeddings of $\mathfrak{A}$ and $\mathfrak{B}$ as subvarieties of $\mathfrak{A}+\mathfrak{B}$. Lawvere has observed ([4] p. 48) that $\mathfrak{A}+\mathfrak{B}$ is the variety of algebras which split naturally as a product $A \times B$ of an $\mathfrak{A}$-algebra by a $B$-algebra. We briefly sketch a proof.

The element $\left(p_{1}, p_{2}\right) \in H(\mathfrak{A}) \times H(\mathfrak{B})$ defines as above an $(\mathfrak{U}+\mathfrak{B})$ operation which is binary, associative, and satisfies the laws $x x=x$ and 
$x y z=x z$. If $C$ is any algebra in $A+B$, consider the relation on $C$ defined by: $c_{1} \equiv c_{2}$ means $c_{1}=c_{2} c_{1}$. This relation is reflexive since $c=c c$, symmetric since $c_{1}=c_{2} c_{1}$ implies $c_{2}=c_{2} c_{2}=c_{2} c_{1} c_{2}=c_{1} c_{2}$, and transitive since $c_{1}=-c_{2} c_{1}$ and $c_{2}=c_{3} c_{2}$ implies $c_{1}=c_{3} c_{2} c_{1}=c_{3} c_{1}$. Thus it is an equivalence, and a little more work, using the way $\left(p_{1}, p_{2}\right)$ combines with the other $(\mathfrak{A}+\mathfrak{B})$-operations, shows that it is even a congruence and that the quotient $A=C / \equiv$ is in the subvariety $\mathfrak{A}$ of $\mathfrak{A}+\mathfrak{B}$. Similarly if $c_{1} \sim c_{2}$ is defined to mean $c_{1}=c_{1} c_{2}$, then the quotient $C / \sim$ is an algebra in the subvariety $B$ of $\mathfrak{A}+\mathfrak{B}$. The projections $p: C \rightarrow A$ and $q: C \rightarrow B$ give a homomorphism $\varphi: C \rightarrow A \times B$ by $\varphi(c)=(p(c), q(c))$. This has an inverse given by $\psi\left(p\left(c_{1}\right), q\left(c_{2}\right)\right)=c_{1} c_{2}\left(c_{1}, c_{2} \in C\right)$, so it is an isomorphism.

Example 6. Given two $\boldsymbol{\aleph}_{0}$-clones $K$ and $L$, one can form the free sum $K+L$. This corresponds to the product $\mathfrak{B}(K) \times \mathfrak{B}(L)$ in the categary $\mathscr{V}$ ar. An example is given by the variety $\mathfrak{M}_{R}$ of $R$-modules, where $R$ is a ring with unity. Let $\mathfrak{A}_{R}$ be the variety of affine $R$-modules (affine spaces over $R$ ) obtained by forgetting all but the idempotent operations from the variety of $R$-modules. Then Satz 1 of [8] states that

$$
\mathfrak{M}_{R}=\mathfrak{A}_{R} \times \mathfrak{\subseteq}_{0},
$$

where $\mathfrak{S}_{0}$ is pointed sets (the variety defined by one constant operation).

An algebra of $\mathfrak{A} \times \mathfrak{B}$ is just a set carrying an $\mathfrak{A}$-algebra structure and a $\mathfrak{B}$-algebra structure, these structures being unrelated by any laws. In view of this, it is surprising that even slightly non-trivial examples occur in "everyday life".

A question which arises in this connection is whether every variety can be split as a product of product-irreducible varieties, and if so, in how far such a splitting is unique. For a full species the obvious splitting into a product of varieties each defined by one operation is the only splitting into product-irreducible varieties, but no other results in this direction seem to be known. The same question can of course be formulated for sums.

To close, we describe an amusing construction which yields varieties which have isomorphic abstract categories, but are not rationally equivalent.

Let $K$ be an $\boldsymbol{\aleph}_{0}$-clone, $n$ a positive integer, and define $K^{[n]}$ to be the $\aleph_{0}$-clone with underlying set $K^{n}$ and structure defined as follows

$$
\begin{aligned}
& p_{i+1}=\left(p_{i n+1}, p_{i n+2}, \cdots, p_{i n+n}\right) \in K^{n} \quad(i=0,1, \cdots), \\
& \left(\left(g_{1}, \cdots, g_{n}\right)\left(f_{1}, \cdots, f_{n}\right)\left(f_{n+1}, \cdots, f_{2 n}\right) \cdots\right) \\
& \quad=\left(\left(g_{1} f_{1} f_{2} \cdots\right),\left(g_{2} f_{1} f_{2} \cdots\right), \cdots,\left(g_{n} f_{1} f_{2} \cdots\right)\right) .
\end{aligned}
$$

It is a simple calculation to show that this gives an $\boldsymbol{\aleph}_{0}$-clone structure. For any variety $\mathfrak{A}$ denote $\mathfrak{B}\left(H(\mathfrak{Q})^{[n]}\right)$ by $\mathfrak{A}^{[n]}$. 
One can show that $\mathfrak{U}^{i{ }^{i j}}$ is the variety of aigebras whose underlying sets can be written canonically as a power $A^{\text {th }}$ of an $\mathfrak{A}$-algebra $A$, and whose homomorphisms are the maps of the form $A^{n} \rightarrow B^{n}$ which are induced by a homomorphism $A \rightarrow B$ of $\mathfrak{U}$-algebras. In particular it follows that $\mathfrak{A}^{[n]}$ has the same underlying category as $\mathscr{U}$. F. W. Lawvere has pointed out in a letter to the author that if $R$ is a ring with unity and $9 \mathcal{M}_{R}$ the variety of $R$-modules, then $\mathfrak{M}_{R}^{n\rfloor}$ is rationally equivalent to the variety $\mathfrak{M}_{R_{n}}$ of $R_{n}$-modules, where $R_{n}$ is the ring of $n$-square matrices over $R$.

It can happen that $\mathfrak{U}^{[n]}$ and $\mathfrak{Q}^{[m]}$ are rationally equivalent with $n \neq m$. However if $\mathfrak{A}$ contains finite non-singular algebras this can clearly not happen, as the smallest non-trivial algebras in $\mathfrak{U}^{[n]}$ and $\mathfrak{U}^{[m]}$ have respectively $k^{n}$ and $k^{m}$ elements, for some $k>1$. Hence in this case one obtains infinitely many varieties having the same underiying category, but no two of which are rationally equivalent.

\section{References}

[1] P. M. Cohn, Universal Algebra (New York, 1965).

[2] E. C. Dale, 'Semigroup and braid representations of varieties of algebras', Thesis, (Manchester 1956).

[3] W. Felscher, 'Equational maps', Contrib. to Math. Logic, (Amsterdam 1968), 121-161.

[4] F. W. Lawvere, 'Some algebraic problems in the context of functorial semantics of algebraic theories', Reports of the Midwest Category Seminar II, (Springer Verlag, 1968), $41-61$.

[5] F. E. J. Linton, 'Some aspects of equational categories', Proc. Conf. Categ. Alg. La Jolla, 1965, (Springer Verlag, 1966), 84-94.

[6] B. H. Neumann and E. C. Wiegold, 'A semigroup representation of varieties of algebras', Coll. Math 14 (1966), 111-114.

[7] W. D. Neumann, 'On cardinalities of free algebras and ranks of operations', Arch. Math. 20 (1969), $132-133$.

[8] F. Ostermann and J. Schmidt, 'Der baryzentrische Kalkül als axiomatische Grundlage der affinen Geometrie' J. reine angew. Maih. 224 (1966), $44-57$.

Mathematisches Institut der

Universität Bonn 